\title{
A Quantitative Description of NMDA Receptor-Channel Kinetic Behavior
}

\author{
Craig E. Jahr ${ }^{1}$ and Charles F. Stevens ${ }^{2, a}$ \\ 'The Vollum Institute, Oregon Health Sciences University, Portland, Oregon 97201, and ${ }^{2}$ The Section of Molecular \\ Neurobiology, Yale University School of Medicine, New Haven, Connecticut 06510
}

\begin{abstract}
Currents evoked in neurons of the vertebrate CNS by the glutamate agonist $\mathrm{N}$-methyl-D-aspartate (NMDA) exhibit a marked voltage dependence in the presence of extracellular Mg. At the single-channel level, the addition of external Mg alters single-channel openings from long-lived events to many very short events grouped into bursts of openings. These bursts apparently result from short interruptions of current flow during periods when the channel is in the open configuration.

Single-channel currents evoked by NMDA have been studied in outside-out patches of membrane taken from hippocampal CA1 neurons grown in dissociated cell culture. The effects of changing external Mg concentration and holding potential on the single-channel parameters of open time, closed time, and burst length have been successfully described assuming a 3- or 4-state model with 1 open state, 1 or 2 "blocked" states, and 1 absorbing closed state. Evaluation of the blocking rates over $\mathrm{Mg}$ concentrations from $0.2-200 \mu \mathrm{M}$ indicate that a single "blocking" mechanism cannot account for the short closed states and that a second voltage-dependent but Mg-independent "blocked" state is necessary to explain the data especially at low Mg concentrations.
\end{abstract}

The excitatory amino acid channels selectively activated by $N$-methyl-D-aspartate (NMDA) are remarkable because their gating is jointly controlled by a neurotransmitter, probably glutamate (Watkins and Evans, 1981; Mayer and Westbrook, 1987), and by a voltage-dependent block of the channel produced by physiological concentrations of Mg ions (Mayer et al., 1984; Nowak et al., 1984). This dual control means that these channels can contribute to the synaptic conductance only if 2 conditions are met: neurotransmitter is released by the presynaptic neuron and, at the same time, the postsynaptic neuron is sufficiently depolarized to relieve the channel block (Collingridge et al., 1988; Forsythe and Westbrook, 1988). The goal of the work described here is, through an analysis of single-channel currents, to provide a quantitative description of the effect of membrane voltage on the blockade of NMDA receptor channels by $\mathrm{Mg}$.

\footnotetext{
Received Sept. 14, 1989; revised Dec. 1, 1989; accepted Dec. 8, 1989.

Supported by NIH grants NS21419 (C.E.J.) and NS12961 (C.F.S.), The McKnigh Endowment Fund for Neuroscience (C.E.J.), and the Howard Hughes Medical Institute (C.F.S.)

Correspondence should be addressed to Craig E. Jahr, Vollum Institute L474 Oregon Health Sciences University, 3181 SW Sam Jackson Park Road, Portland, OR 97201 .

Present address: The Salk Institute, P.O. Box 85800, San Diego, CA 92138. Copyright $(\mathcal{C} 1990$ Society for Neuroscience $0270-6474 / 90 / 061830-08 \$ 02.00 / 0$
}

The ability of $\mathrm{Mg}$ to antagonize the depolarizing action of NMDA had been appreciated for some time (Evans et al., 1977) before it was reported that the voltage dependence of the NMDA response (MacDonald and Wojtowicz, 1982) was conferred by a block of the ionic pore by $\mathrm{Mg}$ (Mayer et al., 1984; Nowak et al., 1984). At physiological $\mathrm{Mg}$ concentrations (near $1 \mathrm{~mm}$ ), NMDA receptor channels do not conduct current if the postsynaptic membrane potential is more negative than about -80 $\mathrm{mV}$, but they conduct current freely if the membrane potential is, for example, $+20 \mathrm{mV}$. This conclusion is based on 2 main observations. First, the voltage dependence, as revealed by negative slope region of the current-voltage relation, is mostly eliminated if $\mathrm{Mg}$ ions are removed from the extracellular solution (Mayer et al., 1984; Nowak et al., 1984). Second, a pattern of brief interruptions of single-channel currents, much like that identified as channel blocking in other systems (Neher and Steinbach, 1978), is observed; the frequency of these interruptions is directly related to $\mathrm{Mg}$ concentration (Nowak et al., 1984; Ascher and Nowak, 1988).

According to the simplest model of channel block, the receptor can exist in 3 states, closed $(C)$, open $(O)$, and blocked (B), that are connected according to the diagram

$$
\mathrm{C} \longrightarrow \mathrm{O} \longrightarrow \mathrm{B}
$$

where the transition from closed to open would require that neurotransmitter had been bound, and the open to blocked transition would involve the voltage-dependent binding of an $\mathrm{Mg}$ ion. This sequential blocking model is at least roughly in accord with single-channel data. As pointed out in the original description of Mg-dependent interruptions (Nowak et al., 1984), however, the model given by scheme (1) predicts that the open burst (in which the channel alternates between open and blocked states) should lengthen in proportion to Mg concentration (Neher, 1983), but it does not (Nowak et al., 1984; Ascher and Nowak, 1988).

Scheme (1) also fails to predict the voltage dependence of the macroscopic currents (Mayer and Westbrook, 1987). This theory predicts that the total length of time the channel remains in the open state during each burst is independent of $\mathrm{Mg}$ concentration. Thus, the total charge transferred into the neuron each time the channel opens from the closed state is independent of blocking. At agonist concentrations where the probability of opening is low, the average current produced by a population of channels, therefore, is unaffected by the blocking, and the average current passed should not decrease with larger hyperpolarizations. Some modification of scheme (1) is thus needed to provide a satisfactory explanation for the voltage-dependent 
gating that arises from a voltage-dependent block of the channel by $\mathrm{Mg}$ ions.

Our final conclusion will be that an Mg-blocking mechanism is indeed a plausible basis for interruptions, but our description will require a rather complicated model. We arrive at this model in 2 steps. The first step is to show that the simplest extension (2) of the blocking model described above

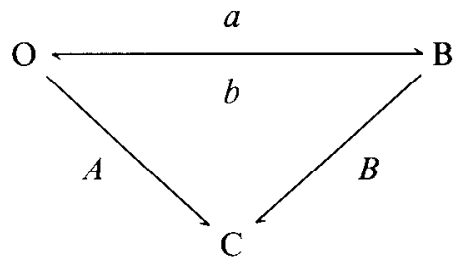

is relatively satisfactory for describing single-channel kinetics at any given $\mathrm{Mg}$ concentration. 'This model, however, is not quite in accord with the physical interpretation of gating as occurring through a voltage-dependent $\mathrm{Mg}$ block. The sccond step is to extend scheme (2) to include 2 distinct blocked states. Although this second model is more complicated in that the channel can occupy 4 (rather than 3 ) states, the complication is necessary to provide an account of channel behavior at all voltages and all $\mathrm{Mg}$ concentrations from $0.2-200 \mu \mathrm{M}$.

\section{Materials and Methods}

Hippocampal neurons from the CA1 region were dissociated from 1to 3-d-old Long-Evans rats. Blocks of tissue from the CAl region of hippocampi were incubated in papain (20 units/ml; Worthington) for $0.5-1.5 \mathrm{hr}$ at $37^{\circ} \mathrm{C}$, with constant agitation. The tissue was twice washed in complete medium [MEM, Earle's salts (Gibco); 20 mM glucose; penicillin, 50 units $/ \mathrm{ml}$; streptomycin, $50 \mathrm{mg} / \mathrm{ml} ; 5 \%$ heat-inactivated fetal bovine serum (Hyclone), Serum Extender (Collaborative Research)] with added BSA ( $5 \mathrm{mg} / \mathrm{ml}$; Sigma) and trypsin inhibitor $(5 \mathrm{mg} / \mathrm{ml}$; Sigma) and then dissociated into a single-cell suspension by trituration with a flamed Pasteur pipette. The cells were plated on glass coverslips coated with collagen/poly-D-lysine (Biomedical Technologies/Collaborative Research). Cultures were fed every $2-4 \mathrm{~d}$ by replacing half the medium. Arabosylcytosine $(5 \mu \mathrm{M})$ was added for $1-2 \mathrm{~d}$ during the first week to suppress proliferation of non-neuronal cells.

Experiments were performed on outside/out patches of membrane (Hamill et al., 1981) taken from neurons grown from 1-3 weeks in culture. Solutions containing NMDA, glycine and different $\mathrm{Mg}$ concentrations were applied by placing the patch inside constantly flowing perfusion tubes to insure complete changes in the concentrations of agonist and $\mathrm{Mg}$. The standard extracellular solution to which NMDA, glycine, and $\mathrm{Mg}$ were added contained (in $\mathrm{mM}$ ): $\mathrm{NaCl}, 165 ; \mathrm{CaCl}_{2}, 2$; HEPES, 5 ( $\mathrm{pH}$ adjusted to 7.3 with $\mathrm{NaOH}$ ). The internal solution usually contained (mM): CsCl, 150; HEPES, 5; EGTA, 10; MgATP, 2 (pH adjusted to 7.3 with $\mathrm{CsOI}$ ). In several experiments the internal solution was altered to determine if internal anions could block the NMDA channel at negative potentials. These solutions contained (in $\mathrm{mm}$ ): (1) Cs gluconate, 140; $\mathrm{CsCl}, 10$; HEPES, 5; EGTA, 10; (2) $\mathrm{CsCl}, 135$; HEPES, 25; EGTA, 10; (3) CsCl, 175; HEPES, 5; EGTA, 1. In all cases, $\mathrm{pH}$ was adjusted to 7.3 with $\mathrm{CsOH}$. The monovalent cation concentrations of the external solution were also altered in several experiments to contain (in mM): (1) NaCl, 486; $\mathrm{CaCl}_{2}, 2$; HEPES, 5 (pH 7.3); (2) $\mathrm{NaCl}, 165 ; \mathrm{CaCl}_{2}, 2 ; \mathrm{HEPES}, 5$ (pH 6.45). $\mathrm{NaCl}, \mathrm{KCl}, \mathrm{CsCl}$, and $\mathrm{CaCl}_{2}$ were purchased from Alfa (Puratronic grade) or Aldrich (Gold Label). Three batches of complete recording medium without added $\mathrm{Mg}$ were assayed (Galbraith Laboratories, Knoxville, TN) and found to have 0.2 $\pm 0.02 \mu \mathrm{M} \mathrm{Mg}$ contamination. Solutions to which no $\mathrm{Mg}$ was added are therefore referred to as having $0.2 \mu \mathrm{M} \mathrm{Mg}$. The agonists used were NMDA (Cambridge Research Biochemicals) plus glycine (BioRad; 2 $\mu \mathrm{M}$ and $1 \mu \mathrm{M}$, respectively) unless otherwise specified. Membrane current was low-pass filtered at $2000 \mathrm{~Hz}(-3 \mathrm{~dB} ; 8$-pole Bessel) and digitally sampled at $100 \mu \mathrm{sec}$ intervals. Dwell times were measured at half amplitude. Only events in the $50 \mathrm{pS}$ size category were included in the analysis. However, subconductance levels (Cull-Candy and Usowicz, 1987; Jahr and Stevens, 1987; Ascher et al., 1988; Cull-Candy et al., 1988 ) occurring during an opening were included in the measured open

\section{Results}

General observations

Our conclusions are based on studies of 24 outside-out patches excised from rat hippocampal neurons from the CA1 field. To avoid problems associated with averaging data from different experiments, we have limited much of our analysis to 2 patches for which we succeeded in collecting data at 4-7 membrane potentials and $3 \mathrm{Mg}$ concentrations. Additional experiments have been carried out, as described below, to check the validity of certain specific points.

At each voltage and each Mg concentration, we collected between 32 and 1073 channel openings (mean, 286) for patches 1 and 2 and up to 7021 openings under a single condition for other patches. For each condition, open and closed time histograms were compiled; typical examples are shown in Figure $1, A, B$. The histograms of dwell times in the closed states clcarly are composed of multiple components. In Figure $1 B$, for example, the closed time histogram for $-60 \mathrm{mV}$ and $10 \mu \mathrm{M} \mathrm{Mg}$ concentration is well fitted with a triple exponential with time constants (and relative amplitudes) of $0.5 \mathrm{msec}(72 \%), 5 \mathrm{msec}$ $(10 \%)$, and $188 \mathrm{msec}(18 \%)$. The magnitude, and to a much lesser extent the time constant, of the fastest component varied with $\mathrm{Mg}$ concentration, but the other components are found to be $\mathrm{Mg}$ independent.

Whenever a channel exhibits such a mixture of brief and long closed times, openings appear in bursts terminated by a long closed interval. An important question is whether these bursts represent a statistical structure beyond the mere presence of short and long intervals. It may be that the occurrence of a short closed interval makes it more likely, for example, that the next closed interval will be short. Alternatively, short and long intervals might be simply intermixed at random with a probability determined by the relative fraction of each duration class. For the data illustrated in Figure $1 B$, for example, a short interval would be followed by another short interval $72 \%$ of the time if intervals are independent. An appropriate test to determine the dependence of one interval on the next is the serial correlogram. If $N$ successive closed intervals are designated by $t_{1}, t_{2}, \ldots t_{j}, \ldots$ $t_{N}$, the serial covariance function is defined to be

$$
C_{k}=\sum_{j=1}^{N-k}\left(t_{j}-T\right)\left(t_{j+k}-T\right) /(N-k)
$$

where $T$ is the mean closed time. Notice that $C_{0}$ is simply the variance of the set of successive closed intervals, and $C_{k}$ estimates the extent to which interval length is felt $k$ intervals later. We use here the serial correlogram $R_{k}$, which is the covariance function normalized by the variance: $R_{k}=C_{k} / C_{0} . R_{0}=1$ and if intervals are independent, then $R_{k}$ should be close to zero for all $k>0$. If long intervals tend to follow other long intervals (and short follow short), $R_{k}$ will be greater than zero, whereas $R_{k}$ will be negative if intervals tend to alternate (long follows short, etc.). The stronger the influence an interval length has on the one $k$ intervals later, the closer $R_{k}$ is to \pm 1 .

Figure $1 C$ presents a serial correlogram for the closed times that gave rise to the histogram in Figure $1 B$ and reveals that intervals are uncorrclated. Intcrvals were found to be uncorrelated in 132 records from 24 patches. This result greatly sim- 
Figure 1. Distributions of singlechannel characteristics evoked by NMDA and glycine. $A$, Cumulative distribution of open durations recorded at

$-60 \mathrm{mV}$ in the presence $2 \mu \mathrm{M}$ NMDA, $1 \mu \mathrm{M}$ glycine, and $10 \mu \mathrm{M} \mathrm{Mg}$. The distribution is fitted with a single exponential with a time constant of $2.5 \mathrm{mscc}$. $B$, Cumulative distribution of closed durations in the same conditions as in $A$. The closed distribution is fitted with a triple exponential with time constants and amplitudes of $0.5 \mathrm{msec} / 72 \%, 5$ $\mathrm{msec} / 9 \%$, and $188 \mathrm{msec} / 18 \%$. C, Serial correlations of successive closed times from the same data set used in $A$ and $B$ showing that the closed durations are uncorrelated. $D$, Distribution of number of interruptions per burst. The distribution is fitted with a theoretical curve generated with Equation (11).
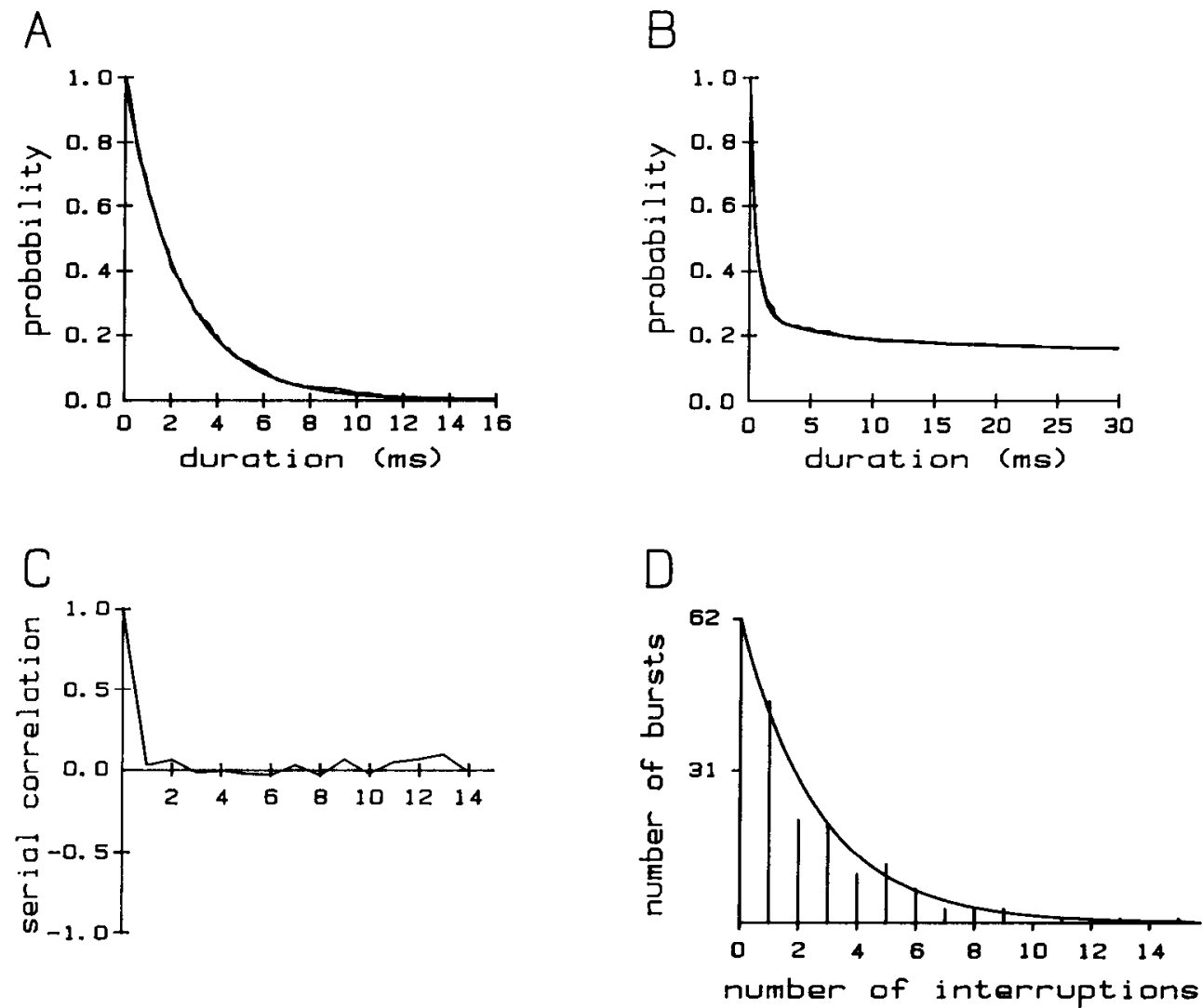

plifies the analysis because models that produce long-range correlations need not be considered. If the closed intervals had been correlated, more than a single open state would be required and the duration of a given closed interval would be influenced by the nature of the preceding open state. Since the closed states were uncorrelated, the assumption of a single open state is sufficient, although not required.

\section{Defining the statistical structure of channel behavior}

Short intervals must arise from a physically distinct mechanism, generally assumed to be $\mathrm{Mg}$ ion block of the pore, because the frequency of their occurrence is dependent on $\mathrm{Mg}$ concentration, whereas that of long intervals is not. Such short closed intervals will be termed interruptions, and the collection of adjaccnt opcnings separated by interruptions are called bursts. We shall need to measure burst length, number of interruptions in each burst, interruption duration, the duration of closed times that are not interruptions, and dwell time in the open state (see Fig. 2 for an illustration of quantities measured). One cannot know definitely whether a particular closing that is, say, $1 \mathrm{msec}$ in duration is an interruption or simply an unusually brief closing of the noninterruption type; that is, we cannot in any individual instance decide whether a brief closed interval is due to an $\mathrm{Mg}$ blocking event or to a closing conformational change succeeded by a rapid reopening. Interruptions can, however, be defined statistically in a consistent way with little error when, as is the case here, their mean duration is much less than the mean duration of the noninterruptions, when interruptions and noninterruption closings are uncorrelated, and when the distribution of interruptions is approximately exponential. The definition of the various intervals is based on the following analysis.
Let $p(t)$ be the probability density for a closed duration $t$, and assume that any given closed time can be due to 1 of 2 distinct mechanisms. Then, $p(t)$ can be written as the sum of 2 components,

$$
p(t)=p_{i}(t)+p_{c}(t)
$$

where $p_{t}$ is the probability density for an interruption and $p_{c}$ is the probability density for the longer, noninterruption closures; in all of our data, $p$, can be fitted with a single exponential with a time constant on the order of $0.5 \mathrm{msec}$, and $p_{c}$ with a multiple exponential, the shortest component of which is an order of magnitude greater than the interruption time constant. If we select a criterion time $t_{\mathrm{cr}}$ defined by the equation

$$
\int_{0}^{t_{\mathrm{cr}}} p_{c}(t) d t=\int_{t_{\mathrm{cr}}}^{\infty} p_{i}(t) d t
$$

and if we call any closed time less than $t_{\mathrm{cr}}$ an interruption, then the number of interruptions that are missed because they are longer than $t_{\mathrm{cr}}$ will be exactly compensated by the number of noninterruption closings that are shorter than $t_{\mathrm{cr}}$ (and are therefore counted, incorrectly, as interruptions). Thus, we will count interruptions correctly. The distribution of interruption durations determined in an experiment, however, will be truncated because no interruptions longer than $t_{\mathrm{cr}}$ are permitted. The mean interruption time will be in error $\left(t_{e}\right)$ by

$$
t_{e}=\int_{t_{\mathrm{cr}}}^{\infty} t p_{i}(t) d t
$$

The fractional error will be $t_{e} / T_{i}$, where $T_{i}$ is the mean inter- 
ruption time. For patches 1 and 2 , the mean critical interval $t_{\mathrm{c}}$ was $2.46 \mathrm{msec}$, the mean interruption time was $0.54 \mathrm{msec}$, and the mean estimated error $t_{e}$ was $0.02 \mathrm{msec}$, or less than $4 \%$ of $T_{i}$. A burst is defined as a series of openings and interruptions terminated by a closure longer than $t_{\text {cr }}$ and has a length given by Equation (7) presented below. The mean error in burst length should be equal to the mean number of interruptions multiplied by the mean error in each interruption length; since channels spend more time open than interrupted except at very negative voltages or very high $\mathrm{Mg}$ concentrations, the mean error in burst time should be less than $2 \%$. Open times are, of course, not influenced by errors in estimating interruption duration, and $t_{\mathrm{cr}}$ is chosen to make the mean number of interruptions per burst accurate. Thus, the quantities that we shall use for our analysis (burst length, interruptions per burst, interruption duration, opcn duration) can all be measured with errors not exceeding $4 \%$. Errors in estimation of various quantities are always comparable to or smaller than sampling errors.

\section{The 3-state model}

The simplest blocking model that can at least qualitatively account for the NMDA receptor-channel gating behavior is illustrated in scheme (2). This model has 4 rate constants $a, b, A$, and $B$ that must be estimated, and we assume that each of these rate constants depends exponentially on membrane potential. That is, any rate constant $R$ has the form

$$
R=\exp \left(S_{R} V+I_{R}\right)
$$

where $R$ represents $a, b, A$, or $B ; V$ is membrane potential; and $S_{R}$ and $I_{R}$ are constants specific for the rate constant in question. The justification for this assumption will be given later.

To compare scheme (2) with experimental observations, we must derive relations between rate constants and experimentally measured quantities. Any burst is characterized by 3 of the 4 quantities: interruption times $\left(t_{i}\right)$, open times $\left(t_{o}\right)$, number of interruptions $(n)$, and burst lengths $\left(t_{b}\right)$. The means of these quantities are related by the equation

$$
T_{b}=(N+1) T_{o}+N T_{i}
$$

where $T_{v}$ and $T_{i}$ are the mean open and interruption times for the burst, $T_{b}$ is the mean burst length, and $N$ is the mean number of interruptions. The primary quantities to be predicted, then, are $N, T_{o}$, and $T_{i}$.

According to scheme (2), $t_{i}$ and $t_{o}$ are exponentially distributed with the form

$$
P_{x}(t)=\exp \left(-t / T_{x}\right)
$$

where $P_{x}(t)$ is the probability of a time $\geq t, x$ is either $i$ or $o$, and the characteristic times $T_{x}$ (which equal the means $T_{i}$ and $T_{o}$ ) are given by

$$
T_{i}=1 /(b+B)
$$

and

$$
T_{o}=1 /(a+A)
$$

The probability of $n$ interruptions per burst, $p(n)$, is, because each interval is independent of the preceding ones, given by

$$
p(n)=q^{n}(1-q)
$$

This is the same equation that, for a coin flipping experiment, specifies the probability of getting $n$ "heads" (interruptions) in a row followed by a "tails" (closure) when the probability of

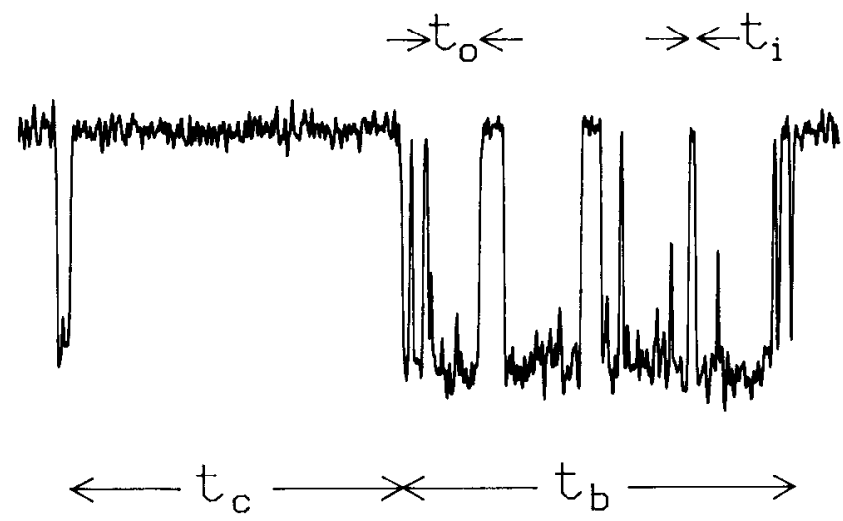

Figure 2. Single-channel parameters measured for analysis. Open duration $\left(t_{o}\right)$, interruption duration $\left(t_{i}\right)$, closed duration $\left(t_{c}\right)$, and burst duration $\left(t_{b}\right)$. Number of interruptions $(n)$ occurring during the burst is 8 .

"heads" is $q$. The mean number of interruptions per burst $(N)$, calculatcd from Equation (10), is

$$
N=q /(1-q)
$$

or

$$
q-N /(N+1)
$$

and $q$ is given by

$$
q=a b /[(a+A)(b+B)]
$$

This last relation gives the probability that, when a transition from the open state occurs, the blocked state, rather than the closed state, will be entered $[a /(a+A)]$ and that the channel will return to the open state from the blocked state $[b /(b+B)]$.

In summary, then, the statistical structure of burst is completely specified, according to this theory, by any 3 of the 4 quantities $T_{b}, T_{i}, T_{o}$, and $N$, and these quantities are in turn related to the 4 rate constants by Equations (7)-(9), (11) and (12), with the rate constants themselves being of the form specified by Equation (6).

\section{Test of the 3-state model}

The first step in testing the 3-state model is to evaluate the adequacy of Equations (8) and (11) for the number of interruptions per burst, the open time, and the interruption time. Insofar as these equations are a satisfactory description of the observed distributions, then we need be concerned only with the mean values of $n, t_{i}$, and $t_{o}$ that completely determine the fit of the theory to experimental data.

Figure $1, A, B, D$, show examples of distributions of $t_{o}, t_{c}$, and $n$ with Equations (8) and (11) superimposed. Clearly, the fits of $t_{o}$ and $n$ in these examples are adequate, and this is true in most instances. Although the distribution of $t_{i}$ cannot be unequivocally separated from $t_{c}$, the time constant of the fastest component of the multiexponential fit will be used to estimate $T_{i}$.

When histograms of $t_{o}$ are constructed from large numbers of events, however, a double-exponential frequently provides a superior fit to the data, especially if the patch was held at positive voltages or in low $\mathrm{Mg}$ concentrations (see Jahr and Stevens, 1987; Cull-Candy et al., 1988; Ascher et al., 1988). Theories like that represented in scheme (2) predict that dwell times should be single exponentials. If more than a single exponential 
Figure 3. Voltage dependence of single-channel parameters, mean open time, mean interruption time, and mean number of interruptions per burst, recorded in $100 \mu \mathrm{M} \mathrm{Mg}$. The theoretical curves were generated using Equations (6), (9), (11), and (12).

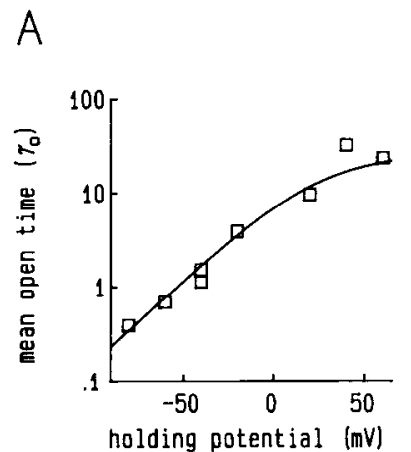

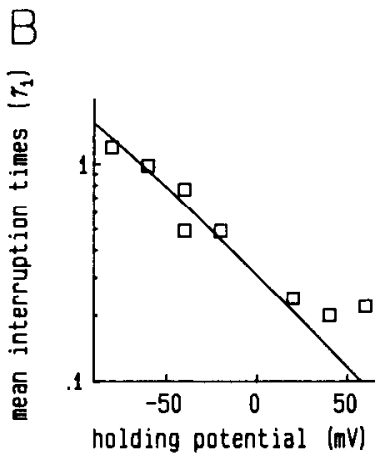

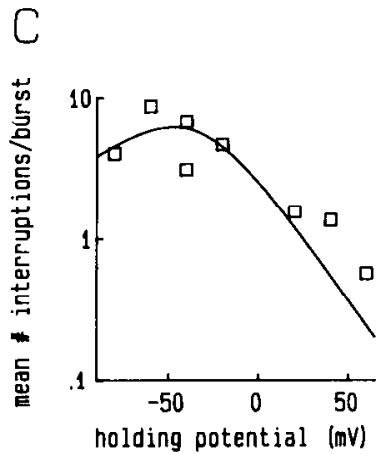

is required to fit dwell time data, one of the assumptions of the model must be incorrect. Possibilities are that transitions do not occur according to a Poisson process, that the channels present do not have homogeneous properties, or that a channel has access to multiple open states that make transitions with somewhat different rate constants. Although we cannot evaluate the first 2 possibilities experimentally, we do have direct evidence for the existence of different open states that could, potentially at least, explain the existence of a multiple exponential open time distribution. Single-channel conductances fluctuate from opening to opening by amounts that exceed the uncertainty in estimating the mean current during an opening, and one occasionally observes transitions between conductance levels of an open channel (Cull-Candy and Usowicz, 1987; Jahr and Stevens, 1987; Ascher et al., 1988). Although events with conductances lower than $45 \mathrm{pS}$ were excluded from this analysis since they clearly can have shorter mean open times (Cull-Candy and Usowicz, 1987; Ascher et al., 1988), we do not know if those events greater than $45 \mathrm{pS}$ which then close to a lower conductance level before completely closing display different kinetics than those that never enter a lower conductance state. Whatever the cause for the multiple exponential open time distributions, the mean open time, $T_{o}$, describes the open time distribution

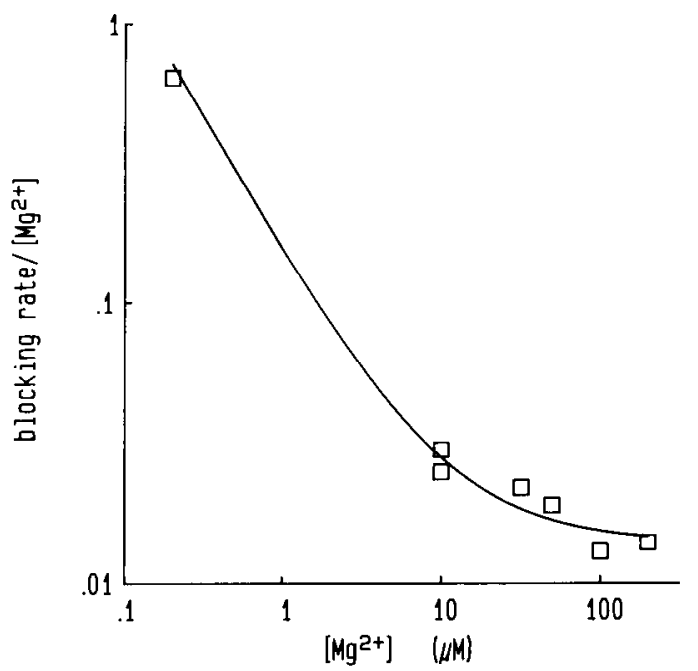

Figure 4. Blocking rate normalized by the $\mathrm{Mg}$ concentration vs $\mathrm{Mg}$ concentration at a holding potential of $-60 \mathrm{mV}$. Note the marked concentration dependence indicating that a single "blocking" mechanism cannot account for all of the interruptions. The data are fitted with the 4-state equation: blocking rate/[ $\left.\mathrm{Mg}^{2+}\right]=\left(a_{1}+a_{2}\right) /\left[\mathrm{Mg}^{2+}\right]$. sufficiently well for the present analysis. A more refined analysis of NMDA receptor channel kinetic behavior would have to address this problem, but the approximate treatment used can be claimed to capture the main effects if the theory is successful.

In summary, then, the predictions of the 3-state model for the distributions of open times, interruption times, and number of intcrruptions per burst [Eqs. (8), (11)] are in at least approximate agreement with our observations. To the extent that this agreement is satisfactory, the 3-state model can be tested by comparing predicted and observed average values for open time, interruption time, and number of interruptions per burst as a function of voltage and for various $\mathrm{Mg}$ concentrations. Specifically, as the second step in testing the 3-state model, we need to evaluate Equations (9) and (11) with rate constants of the form given in (6). For patches 1 and 2, we collected singlechannel currents at $6 \mathrm{Mg}$ concentrations $(0.2,10,32,50,100$, and $200 \mu \mathrm{M}$ ), and at 4-7 voltages from -80 to $+60 \mathrm{mV}$. We have used a straightforward least-squares method to give an estimate of the rate constants in scheme (2).

For each $\mathrm{Mg}$ concentration, we estimated $S_{x}$ and $I_{x}$ in Equation (6) for each of the 4 rate constants in scheme (2). This was done by substituting the expression for the rate constants [Eq. (6) into Eqs. (9ab) and (12)] and minimizing the sum of squared differences between the predictions of the equations and the observed values for $T_{o}, T_{i}$, and $N$ at the various voltages used. At every $\mathrm{Mg}$ concentration the voltage dependence of the closing rate constants $A$ and $B$ were close to zero and of either sign. These values were thus constrained to zero and the rate constants redetermined.

The kinetic model described in scheme (2) proved generally satisfactory in describing the observed averages for $T_{o}, T_{i}$, and $N$. A comparison of the predicted and observed mean values is shown for one $\mathrm{Mg}$ concentration $(0.1 \mathrm{~mm})$ in Figure 3; other fits are about as satisfactory. Although scheme (2) can fit our data for any given $\mathrm{Mg}$ concentration, no single set of rate constants can describe the channel behavior for the entire range of voltages and $\mathrm{Mg}$ concentrations. In fact, the physical interpretation of interruptions as described by scheme (2) cannot be correct. According to the $\mathrm{Mg}$ blocking interpretation of the rate constant $a$ in scheme (2), mass action requires that the blocking rate should depend linearly on $\mathrm{Mg}$ concentration (or, more properly, on $\mathrm{Mg}$ activity). To evaluate this prediction, we have estimated the empirical $\mathrm{Mg}$ blocking rate $r$ per $\mathrm{mm}$ of $\mathrm{Mg}$ present (for fixed voltage) using the equation

$$
r=N /\left[c\left(T_{b}-N T_{i}\right)\right]
$$

Here, $r$ is the blocking rate/mM, $c$ is the $\mathrm{Mg}$ concentration, and 


\section{Table 1}

Rate constants

$a_{1}=\exp (-0.016 \mathrm{~V}-2.91) \mathrm{msec}^{-1}$

$a_{2}=C \exp (-0.045 \mathrm{~V}-6.97) \mu \mathrm{M}^{-1} \mathrm{msec}^{-1}$

$b_{1}=\exp (0.009 \mathrm{~V}+1.22) \mathrm{msec}^{-1}$

$b_{2}=\exp (0.017 \mathrm{~V}+0.96) \mathrm{msec}^{-1}$

$A=\exp (-2.847) \mathrm{msec}^{-1}$

$B_{1}=\exp (-0.693) \mathrm{msec}^{-1}$

$B_{2}=\exp (-3.101) \mathrm{msec}^{-1}$

the other symbols are as previously defincd. The denominator of this fraction gives the mean total open time in each burst so that the ratio estimates the number of interruptions per msec of time available for an interruption to occur and per mм of Mg present to block. The rate constant $r$ is determined for each $\mathrm{Mg}$ concentration (at a fixed voltage $=-60 \mathrm{mV}$ ) and is plotted as a function of $\mathrm{Mg}$ concentration. If $r$ arose from a blocking mechanism and if scheme (2) were correct, $r$ should be independent of $\mathrm{Mg}$ concentration.

As can be seen from Figure 4, $r$ estimated in this way is not independent of $\mathrm{Mg}$ concentration, but rather increases dramatically with decreasing $\mathrm{Mg}$ concentration. $r$ would become independent of $\mathrm{Mg}$ concentration if all of our solutions were contaminated by $15-20 \mu \mathrm{M} \mathrm{Mg}$. This is unlikely, however, since we used very pure salts and determined the $\mathrm{Mg}$ concentration in our nominally $\mathrm{Mg}$-free solutions to be $0.2 \mu \mathrm{M}$. Thus, in spite of the fact that scheme (2) provides a satisfactory formal description of channel behavior at any single $\mathrm{Mg}$ concentration used, either the physical interpretation of $a$ as the rate of block by $\mathrm{Mg}$ ions is incorrect or scheme (2) is an insufficiently accurate description of the channel's function.

\section{The 4-state model}

Although the 3-state model cannot actually be correct, its formal success suggests a simple modification that maintains the notion that interruptions do arise by a $\mathrm{Mg}$ block. Because interruptions still occur at very low $\mathrm{Mg}$ concentrations, an obvious possibility is that a second blocking mechanism is present in addition to the $\mathrm{Mg}$ block. The appropriate state diagram is

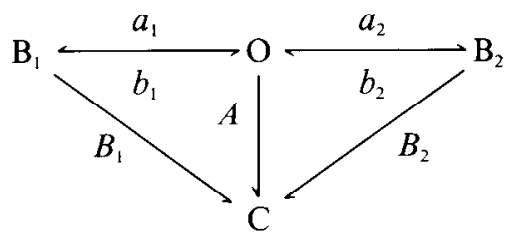

where $B_{1}$ would be the alternative blocked state and $B_{2}$ the $\mathrm{Mg}$ block state. The rate constants are, as indicated in (13), $a_{1}, a_{2}$, $b_{1}, b_{2}, A, B_{1}$, and $B_{2}$. Note that, for the proposed physical interpretation of (13), $a_{1}$ is independent of $\mathrm{Mg}$ concentration and $a_{2}$ depends linearly on $\mathrm{Mg}$. A least-squares procedure estimated these rate constants using data from all voltages and $\mathrm{Mg}$ concentrations and gave the values presented in Table 1. Figure 5 shows the comparison of predicted and observed data for the range of $\mathrm{Mg}$ concentrations and voltages used in our experiments. The fit reveals that the 4-state model provides a satisfactory description of the data (see Appendix).
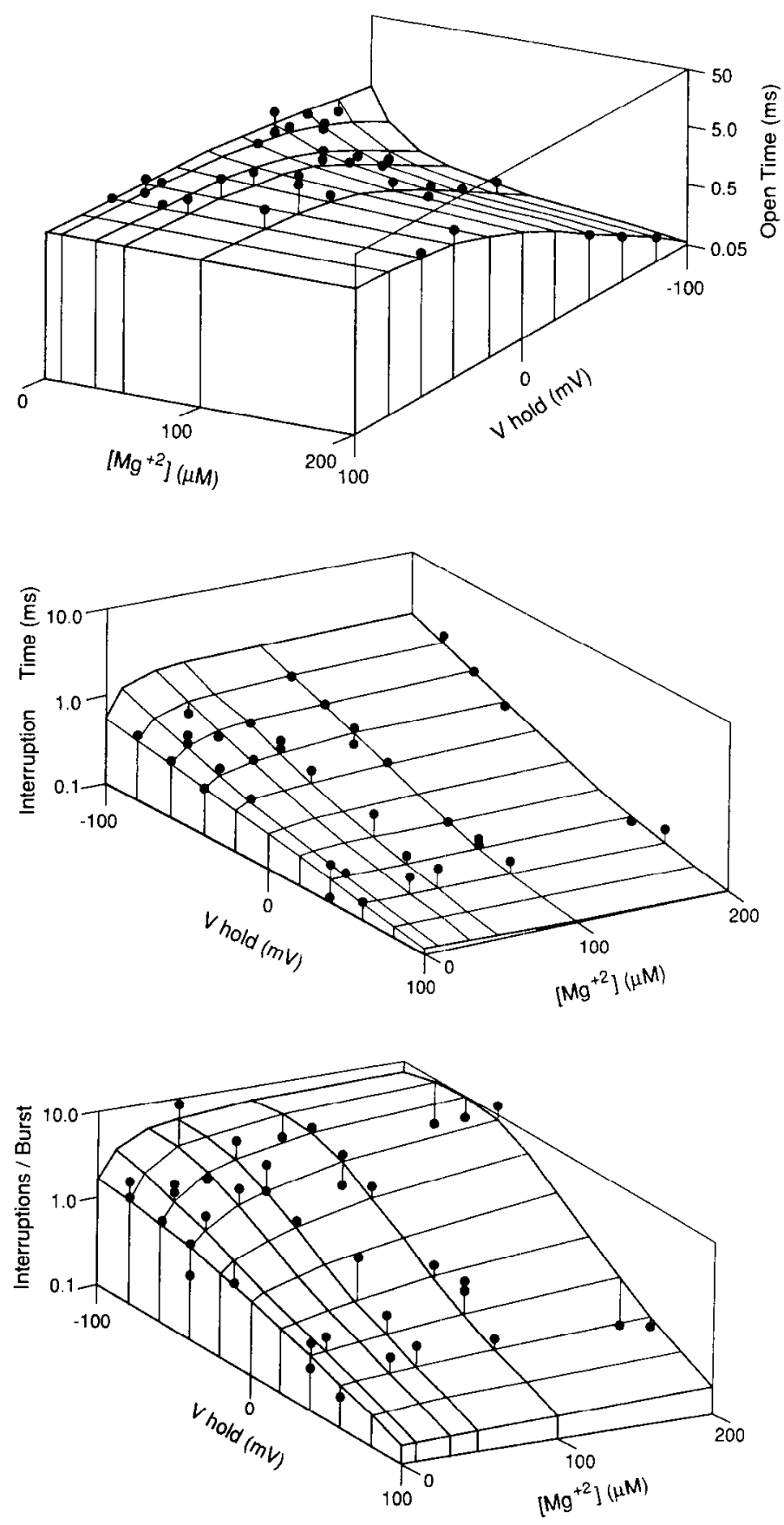

Figure 5. Open duration, interruption duration, and number of interruptions/burst vs voltage and $\mathrm{Mg}$ concentration. The theoretical surfaces are generated from the 4-state model using Equations (6), (9), (11), and (12) after substitution of the 4-state rate constants given in the Appendix.

If the interruptions seen at low $\mathrm{Mg}$ concentrations are not due to $\mathrm{Mg}$, are they perhaps blocking events by some other ion? Note that the voltage dependence of the interruption rate in low $\mathrm{Mg}$ is less than half of that at higher $\mathrm{Mg}$ concentrations (compare $a_{1}$ and $a_{2}$ in Table 1). This suggests that the interruptions in the absence of $\mathrm{Mg}$ have a different mechanism, perhaps the result of a monovalent ion block. We have changed sodium, $\mathrm{pH}$, and calcium in the extracellular solution (the only cations present), and chloride, EGTA, and HEPES in the internal solution and have found no change in the rate of interruptions in nominally 
7ero (actually $0.2 \mu \mathrm{M}$ ) Mg. Specifically, decreasing $\mathrm{pH}$ from 7.4 to 6.5 or increasing sodium ion concentration from 160 to 486 mM produced no change in $T_{b}, T_{i}$, or $N$. In the high sodium concentration solution, the single-channel conductance increased from 50 to $70 \mathrm{pS}$. Increasing calcium concentration from 1 to $10 \mathrm{~mm}$ similarly caused no increase in interruptions, although the single-channel conductance was decreased to about half the normal value. No change in interruption rate was observed when internal ionic constituents were individually altered (chloride from 150 to $10 \mathrm{~mm}$, HEPES from 5 to $25 \mathrm{~mm}$, and EGTA from 10 to $1 \mathrm{~mm}$ ). Furthermore, the interruption rate seems not to vary with the concentration of NMDA (0.5$10 \mu \mathrm{M})$ used to open channels. If the interruptions seen at low $\mathrm{Mg}$ concentrations represent a channel blocking event by an ion, we have not been able to identify the blocking ion.

\section{Discussion}

Ascher and Nowak (1988) have examined the question of $\mathrm{Mg}$ block using methods similar to those employed here. Their results are qualitatively similar to the ones we have presented, but they did not pursue a detailed kinetic analysis to fit all the observations. They conclude that a voltage-dependent $\mathrm{Mg}$ block is a likely mechanism for the interruptions and note that $T_{b}$ docs not increase directly with $\mathrm{Mg}$ concentration so that the simple sequential block model (scheme 1) cannot be correct. The voltage dependencies of our blocking and unblocking rates derived from the 3-state model (scheme 2 ) and theirs are quite close: they found that the blocking rate constant $\left(k_{*}\right.$, in their teminology) was $6.1 \times 10^{\mathrm{s}} \exp (-V / 17) \mathrm{M}^{1} \mathrm{sec}^{-1}$ and the unblocking rate $\left(k\right.$ ) was $5.4 \times 10^{3} \exp (V / 47) \mathrm{sec}^{-1}$. Our corresponding values (in $0.2 \mathrm{~mm} \mathrm{Mg}$ ) are $1.06 \times 10^{6} \mathrm{exp}(-V / 21)$ $\mathrm{M}^{1} \mathrm{sec}$ and $2.36 \times 10^{3} \mathrm{exp}(V / 50) \mathrm{sec}^{-1}$. The differences can be ascribed, at least in part, to temperature. As described above, our 3-state rate constants also depend on $\mathrm{Mg}$ concentration because, as determined with the 4-state model, the proportion of time the channel stays in $\mathrm{B} 1$ relative to $\mathrm{B} 2$ is dependent on $\mathrm{Mg}$ concentration; this makes a strict comparison of the results difficult. They do report interruptions in nominally zero $\mathrm{Mg}$ solutions, but these closures were independent of voltage as well. At $-60 \mathrm{mV}$ the mean $K_{c /}$ for $\mathrm{Mg}$ determined from the microscopic rate constants was $73 \mu \mathrm{M}$, which is the same as the 72 $\mu \mathrm{M}$ given by Ascher and Nowak (1988). At $0 \mathrm{mV}$, however, our results diverge; $1.8 \mathrm{~mm}$ in our hands, $8.8 \mathrm{~mm}$ in theirs.

According to scheme 2 , the constant $S_{k}$ (where $R$ is $a_{2}$ or $b_{2}$ here) in Equation (6) would (if the cntire voltage dependence of blocking arises from the movement of $\mathrm{Mg}$ ions through the membrane ficld over a single energy barrier to a single binding site) be

$$
S_{R}-f_{R}=F / R T
$$

where $f_{R}$ is the fraction of the membrane field through which the ion must step to reach the barrier peak, $z=2$ (the valence of $\mathrm{Mg}$ ) and $F / R T=25.4 \mathrm{mV}$ at room temperature ( $F$ is the Faraday constant; $R$, the gas constant, and $T$, the temperature in Kelvin). Solving this equation for $f_{k}$ and using the values of $S_{a_{2}}$ and $S_{h_{2}}$ from Table 1. we find from $S_{a_{2}}$ that an ion on the outside must step through 0.57 of the membrane field to reach the barrier peak and then, from $S_{h_{2}}$, through 0.22 of the membrane field to reach the binding site. The binding site is then about $80 \%$ of the way through the membrane field.

$W_{c}$ have shown that the 3-state model is adequate for higher $\mathrm{Mg}$ concentrations, and that the 4-state scheme will describe all of our results except the low-amplitude second-exponential component in the open time distributions which occurs in low $\mathrm{Mg}$ concentrations and at positive voltages. We turn now to a physical interpretation of these formal kinetic schemes.

Two different interpretations of our results are possible. First (Thcory 1), we might suppose that interruptions mainly result from $\mathrm{Mg}$ ions that enter the pore and block it. On this theory, the voltage dependence of the interruption rate and the duration of interruptions arises because the $\mathrm{Mg}$ ions must pass part way through the membrane field to arrive at their blocking site. This blocking site could be within the pore itself. On this theory, the channel must be able to close with a $\mathrm{Mg}$ ion in the blocking site, and we must suppose that some other mechanism, perhaps a conformational change, is responsible for interruptions in the absence of $\mathrm{Mg}$ ions.

Second (Theory 2), the interruptions could be the result of a voltage-dependent conformational change. In this case, the effect of $\mathrm{Mg}$ ions would be explained by supposing that they must bind at some site to facilitate the conformational change. The picture here would be much like that for the calcium-activated potassium channel where calcium shifts the gating function along the voltage axis, but the voltage dependence is thought to reside in the protein conformational change rather than derive from calcium ions moving through the membrane field in order to reach their binding site (Magleby and Pallotta, 1983: although see Moczydlowski and Latorre, 1983).

Both theories are consistent with the 4-state model presented above, but the second theory accommodates the Mg-independent interruptions more easily. Similar interruptions, however, have long been known to occur in situations where no blocking entity can be identified. Fven when a channel block does occur, as with local anesthetic block of the $\mathrm{ACh}$ receptor channel, for example (Neher and Steinbach, 1978; Neher, 1983), what are apparently nonblock interruptions also occur. Thus, the presence of interruptions without an identified blocker need not be taken to strongly favor the first theory.

The crucial distinction between the 2 theories is that the postulated binding site that facilitates a conformational change in Theory 2 should saturate for sufficiently high $\mathrm{Mg}$ concentrations, whereas Theory 1 predicts that blocking rates increase linearly, without limit, as $\mathrm{Mg}$ concentration is increased. If Theory 1 is correct, the effect of any $\mathrm{Mg}$ concentration can be counteracted with sufficiently large voltage.

The fact that blocking rate increases linearly, at a given voltage, with $\mathrm{Mg}$ concentration favors Theory 1 , but not in a definitive way. Even if Theory 2 were correct, it might be expected that the effect of $\mathrm{Mg}$ would be linear over a narrow range, and the single-channel recording experiments are possible only up to about $0.2 \mathrm{~mm} \mathrm{Mg}$, about one-fifth of physiological concentrations, so the saturation of a binding site might well not be detected at the concentrations we have used here. In order to distinguish between the 2 theories, one would have to increase $\mathrm{Mg}$ concentrations into the range where the Theory $2 \mathrm{Mg}$ binding site might be expected to be blocked. Unfortunately, singlechannel observations are not feasible at $\mathrm{Mg}$ concentrations much above the $0.2 \mathrm{~mm}$ we have used because the interruption rate becomes so great that the brief openings cannot be measured with sufficient accuracy.

In summary, our data are consistent with either physical interpretation, and we cannot, with information at hand, strongly favor one theory over the other. We can conclude, however, that the 3 - and 4-state models described above provide, within 
experimental error, an accurate account of the single-channel behavior.

\section{Appendix}

The 4-state model (13) is a simple extension of the 3-state scheme (2). In this appendix we give the equations relating the mean open time, mean interruption time, and mean number of interruptions per burst for the 2 models.

The rate of leaving the open state in the 3-state model is $a$ and the corresponding rate for the 4-state model is $a_{1}+a_{2}$, the sum of the leaving rate for the 2 states ( 1 and 2 ). The transition rate from blocked to open is $b$ for the 3-state model, whereas the corresponding rate for the 4-state model is $b_{1} a_{1} /\left(a_{1}+a_{2}\right)+b_{2} a_{2} /\left(a_{1}+a_{2}\right)$; in this last expression, the rate of leaving each blocked state is weighted by the probability that the particular state had been entered. The closing rate from the blocked state is $B$ for the 3 -state model and $B_{1} a_{1} /\left(a_{1}+a_{2}\right)+B_{2} a_{2} /\left(a_{1}+a_{2}\right)$ for the 4-state model. If the following equations are made

$$
\begin{aligned}
a & =a_{1}+a_{2} \\
b & =\left(b_{1} a_{1}+b_{2} a_{2}\right) /\left(a_{1}+a_{2}\right) \\
A & =A
\end{aligned}
$$

and

$$
B=\left(B_{1} a_{1}+B_{2} a_{2}\right) /\left(a_{1}+a_{2}\right)
$$

then the mean open time $\left(T_{o}\right)$ and mean interruption time $\left(T_{i}\right)$ given by (9) will be the same for both models.

The open time distribution will be a single exponential with a time constant $T_{o}$ for both models as given by $(8)$; the interruption time dis* tribution will, however, be a double exponential with time constants $1 /\left(b_{1}+B_{1}\right)$ and $1 /\left(b_{2}+B_{2}\right)$ and relative amplitudes $b_{1} / b$ and $b_{2} / b$ for the 4-state model, rather than the single-exponential distribution for the 3 -state model [Eq. (8)]. If the 2 time constants for the 4-state model interruption distribution are not widely separated, then the 2-time-constant distribution would be very hard to distinguish from the single time constant distribution predicted by the 3-state model. If, on the other hand, the 2 time constants were widely separated, either the faster one would be too rapid to be resolved or the longer one would appear to be a closure, and again the interruption times would seem to be distributed according to Equation (8). The interruption time distribution, then, is unlikely to be helpful in distinguishing between the 2 models.

The mean number of interruptions per burst $N$ is related in Equation (11) to the probability $q$ that a blocked state will be entered when the open state is left; then $1-q$ is the probability that, when a transition out of the open state occurs, the channel will enter the closed state without reopening. With the identification given in (A1), Equation (12) is correct for the 4 -state model because $a^{\prime}(a+A)$ is the probability that a transition out of the open state will be into a blocked state and $b /(b$ $+B)$ is the probability that the open state will be reentered when the blocked state is left. The distribution of number of openings per burst [Eq. (1)] is therefore the same for both the 3-and 4-state models.

The 3- and 4-state models are thus formally equivalent for a fixed set of rate constants except for the interruption time distribution, and limitations in the time resolution inherent in our system make it unlikely that this distribution can be used to distinguish between the 2 models. The only practical way of deciding which model to prefer is to change $\mathrm{Mg}$ concentration and voltage and then to examine the dependence of predicted quantities on these variables as we have described in the text.

\section{References}

Ascher P, Nowak L (1988) The role of divalent cations in the $\mathrm{N}$-methyl-D-aspartate responses of mouse central neurones in culture. J Physiol (Lond) 399:247-266.

Ascher P, Bregestovski P, Nowak L (1988) N-methyl-D-aspartateactivatcd channcls of mouse central ncuroncs in magnesium-free solutions. J Physiol (Lond) 399:207-226.

Collingridge GL, Herron CE, Lester RAJ (1988) Synaptic activation of N-methyl-D-aspartate receptors in the Schaffer collateral-commissural pathway of rat hippocampus. J Physiol (Lond) 399:283-300.

Cull-Candy SG, Usowicz MM (1987) Multiple-conductance channels activated by excitatory amino acids in cultured cerebellar neurones. Nature 325:527-528.

Cull-Candy SG, Howe JR, Ogden DC (1988) Noise and single channels activated by excitatory amino acids in rat cerebellar granule neurones. J Physiol (Lond) 400:189-222.

Evans RH, Francis AA, Watkins JC (1977) Selective antagonism by $\mathrm{Mg}^{2+}$ of amino acid-induced depolarizations of spinal neurones. Experientia 33:489-491.

Forsythc ID, Wcstbrook GL (1988) Slow cxcitatory postsynaptic currents meditated by $\mathrm{N}$-methyl-D-aspartate receptors on cultured mouse central neurones. J Physiol (Lond) 396:515-533.

Hamill OP, Marty A, Neher E, Sakmann B, Sigworth FJ (1981) Improved patch-clamp techniques for high resolution current recording from cells and cell-free membrane patches. Pfluegers Arch 391:85100.

Jahr CE, Stevens CF (1987) Glutamate activates multiple single channel conductances in hippocampal neurones. Nature 325:522-525.

MacDonald JF, Wojtowicz JM (1982) The effects of L-glutamate and its analogues upon the membrane conductance of central murine neurones in culture. Can J Physiol 60:282-296.

Magleby KL, Pallotta BS (1983) Calcium dependence of open and short interval distributions from calcium-activated potassium channcls in culturcd rat musclc. J Physiol (Lond) 344:585-604.

Mayer ML, Westbrook GL, Guthrie PB (1984) Voltage-dependent block by $\mathrm{Mg}^{2+}$ of NMDA responses in spinal cord neurones. Nature 309:261-263.

Mayer ML, Westbrook GL (1987) The physiology of excitatory amino acids in the vertebrate central nervous system. Prog Neurobiol 28: $197-276$

Moczydlowski E, Latorre R (1983) Gating kinetics of $\mathrm{Ca}^{2+}$-activated $\mathrm{K}^{+}$channels from rat muscle incorporated into planar lipid bilayers. $\mathrm{J}$ Gen Physiol 82:511-542.

Neher E, Steinbach JH (1978) Local anaesthetics transiently block currents through single acetylcholine-receptor channels. J Physiol (Lond) 277:153-176.

Neher E (1983) The charge carried by single-channel currents of rat cultured muscle cells in the presence of local anesthetics. J Physiol (Lond) 339:663-678.

Nowak L, Bregestovski P, Ascher P, Herbet A, Prochiantz A (1984) Magnesium gates glutamate-activated channels in mouse central neurones. Nature 307:462-465.

Watkins JC, Evans RH (1981) Excitatory amino acid transmitters. Annu Rev Pharmacol Toxicol 21:165-204. 